\title{
Novel P-in-N Si-Sensor technology for high resolution and high repetition-rate experiments at accelerator facilities
}

\author{
Meghana Mahaveer Patil*, Michele Caselle, Lorenzo Rota \\ Marta Baselga Bacardit, Gudrun Niehues, Erik Bründermann, Marc Weber, \\ Anke-Susanne Müller \\ Karlsruhe Institute of Technology, Germany \\ E-mail: meghana.patilakit.edu
}

\section{Giacomo Borghi, Maurizio Boscardin}

Fondazione Bruno Kessler, Italy

\begin{abstract}
Linear array detectors with high spatial resolution and $\mathrm{MHz}$ frame-rates are essential for high-rate experiments at accelerator facilities. KALYPSO, a line array detector with 1024 pixels operating over 1 Mfps has been developed. To improve the spatial resolution and sensitivity at different wavelengths, novel p-in-n Si microstrip sensors based on have been developed with a pitch of 25 $\mu \mathrm{m}$. The efficiency of the sensor has been improved with the use of anti reflecting (AR) coating layers optimized for near infrared, visible and near ultraviolet. In this contribution the detector system and the sensors will be presented.
\end{abstract}

Topical Workshop on Electronics for Particle Physics (TWEPP2018)

17-21 September 2018

Antwerp, Belgium

\footnotetext{
* Speaker.

${ }^{\dagger}$ now at SLAC National Accelerator Laboratory
} 


\section{Introduction}

KALYPSO is a linear array detector system developed for high-rate experiments at synchrotron light sources and accelerator facilities. The detector system is now employed in different experimental stations requiring high resolution, single-shot measurements at $\mathrm{MHz}$ rates and different wavelengths. One of the main application of such a detector is electro-optical spectral decoding which allows scientists to probe the structure of electron bunches in a non-destructive way and with femtosecond time resolution. The previous version of KALYPSO [1] has been installed in various accelerator facilities, and has provided promising results [2]. The detector consists of a linear array sensor connected to a readout ASIC through high-density wire-bonding interconnections. Novel Si micro-strip sensors with a pitch down to $25 \mu \mathrm{m}$ have been designed at KIT and fabricated by Fondazione Bruno Kessler (FBK). To optimize the sensitivity of the detector at different wavelengths namely the visible, near infra-red (NIR) and near ultra-violet (NUV), different anti-reflective coating (ARC) layers have been integrated to the sensor. The current version of KALYPSO consists of a Si sensor with 1024 pixels connected to eight GOTTHARD ASIC readouts. The outputs of the GOTTHARD readouts are divided among two ADCs.

\section{Fine pitch linear array sensor}

High spatial resolution is fundamental for resolving the electron bunches with ps-fs time resolution. Here at KIT, a silicon sensor with a pitch of $25 \mu \mathrm{m}$ have been designed and optimized to match the spatial resolution requirement.
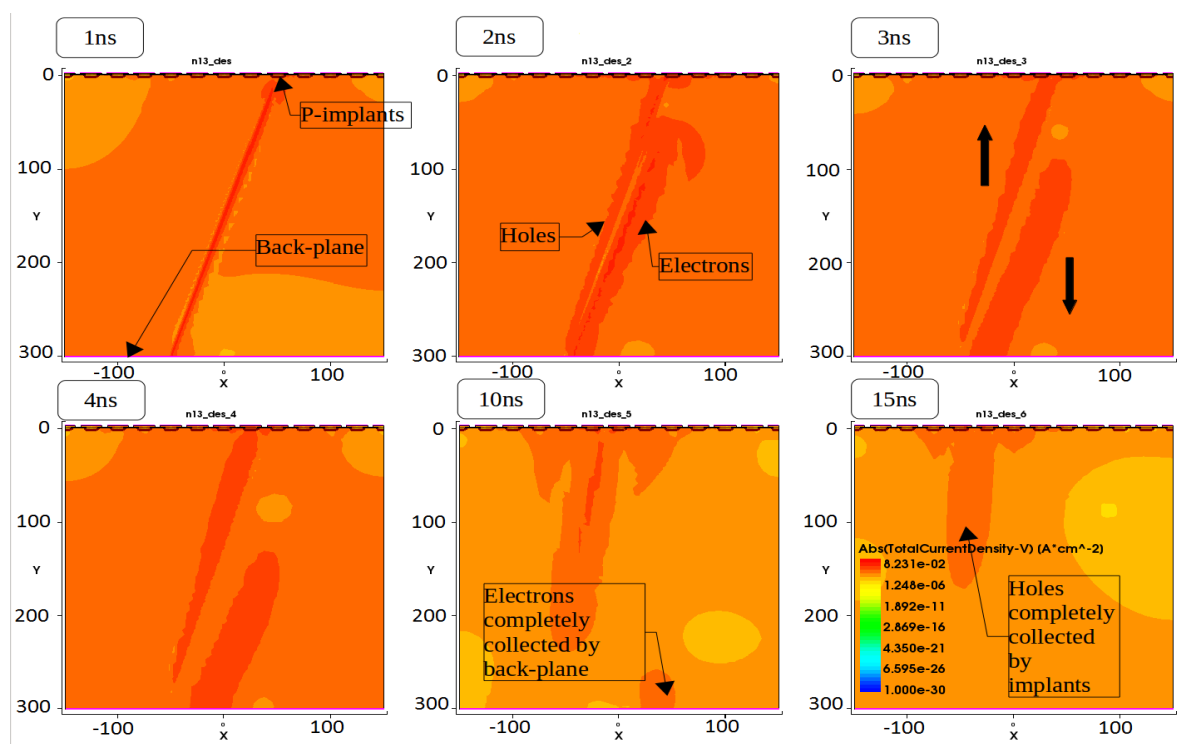

Figure 1: Total current density for a beam of photons at wavelength $1050 \mathrm{~nm}$ incident on the sensor from the backplane. At [1ns] the photon beam penetrates the sensor completely and generates e-/h+ pairs, at [2ns] the e- and $\mathrm{h}+$ start to separate, at [3ns] the separation continues due to the strong electric field, at [10 ns] the electrons are completely collected by the backplane, at [15ns] most of the holes are collected by the strips. 
A Sentaurus TCAD simulation was performed to predict and optimize the electrical behavior of the proposed sensor structures. The Figure 1 is the simulation of the total charge (holes and electrons) collection time for a sensor section for a bias voltage of 100 Volts. The readout pitch on the front end chip is fixed to $50 \mu \mathrm{m}$, hence in order to adapt to this pitch, an interleaved sensor to ASIC integration is required, see Figure 2a. In Figure 2b, the completed layout of the sensor with the interleaved structure is shown. The layout was created using Klayout editor. This editor has a feature which enables the designer to program the structures of the sensor. This makes it easier to parameterize the entire design.

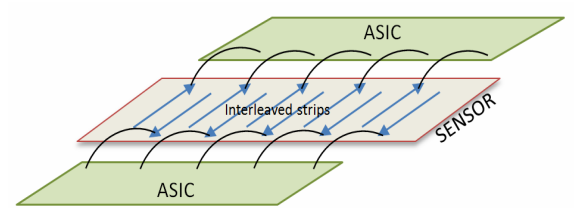

a.

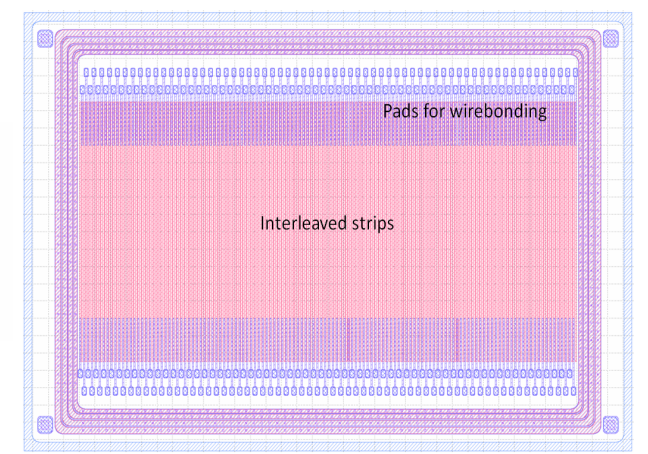

Figure 2: a) Interleaved structure of the sensor,b) Sensor layout for the interleaved structure

Several sensor structures with a pitch of $25 \mu \mathrm{m}$ in large array of sizes 512, 1024 and 2048 has been produced. In addition, other structures with a pitch of $45 \mu \mathrm{m}$ have been produced for an advanced interconnection method based on bump-bonding interconnection technology. The bumpbonding process is a thermo-compression process where two dies are electrically and mechanically connected together [3]. In the proposed scheme, the readout chip is flipped and then connected to the sensor by a so-called "gold stud-ball" bump-bonding process, see Figure 3 and Figure 4. The main advantage such a method is compactness, and reduced noised due to short connections.

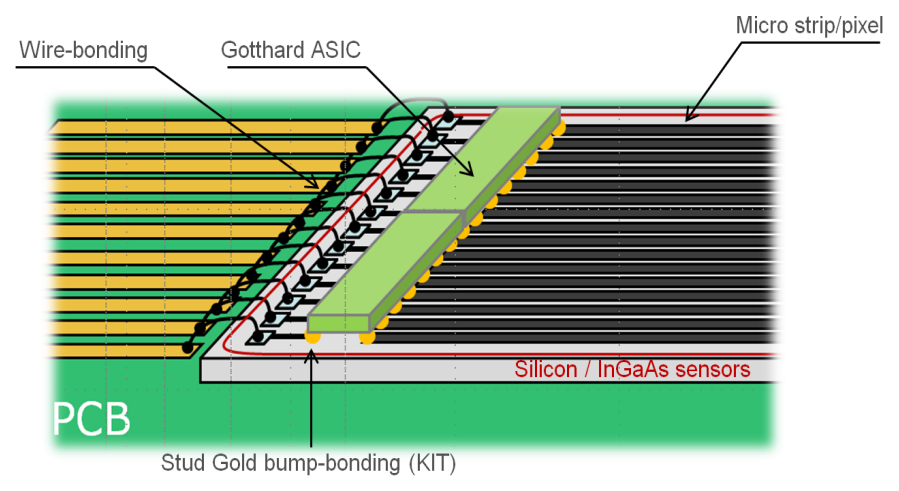

Figure 3: Bump bonding structure for sensor and ASIC integration

Semiconductor materials such as GaAs and $\mathrm{Si}$ are known to have high reflectivity of about 30 to $40 \%$. To reduce reflectivity and enhance sensor efficiency, an anti-reflection (AR) coating layer should be deposited on the sensor surface. AR coatings have been studied in various optical fields 


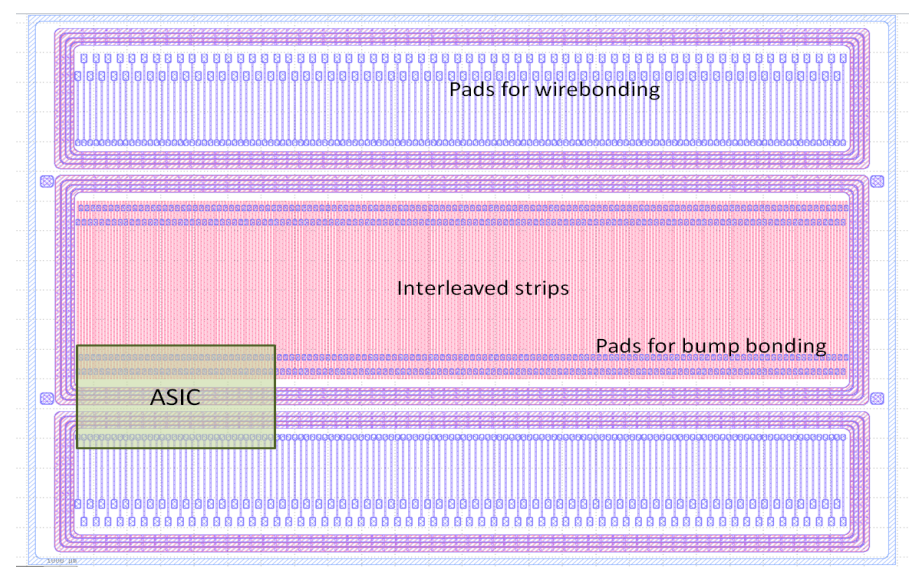

Figure 4: Sensor layout with pads for bump bonding

and are known for increasing transmittance and decreasing reflectance [4]. Hence in order to increase the efficiency of the sensors towards near infrared, visible and near ultra-violet wavelengths, AR coatings layers have been applied to the wafers respectively. Figure 5 shows one such wafer which has been optimized for near infrared wavelength.

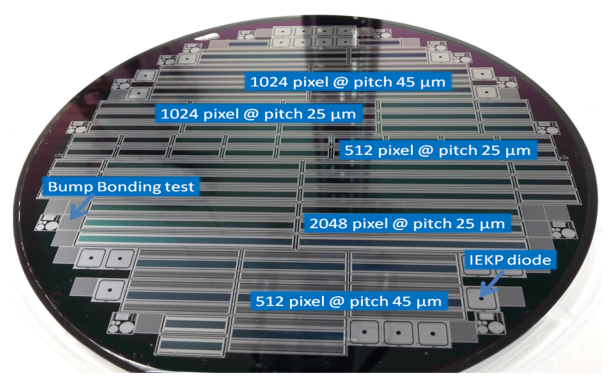

Figure 5: The Silicon wafer optimized for near infrared

\section{Detector Architecture}

The current KALYPSO system contains a line-array of up to 1024 microstrip sensors. This design as shown in the Figure 6 consists of eight readout ASICs. The front-end chip is a modified version of the Gotthard chip. The ASIC, designed in IBM $130 \mathrm{~nm}$ CMOS technology, takes advantage of its three gain stages with automatic stage selection to achieve a dynamic range of 1000012 $\mathrm{keV}$ photons and a noise better than 300 e.n.c.. The 4 analog outputs of the ASIC are optimized for speed, allowing frame rates higher than $1 \mathrm{MHz}$, without compromises on linearity and noise performances [5]. The analog outputs are digitized by a commercial ADC with a sampling rate of $4160 \mathrm{MS} / \mathrm{s}$. In addition to these, the PCB consists of DACs to provide voltage/current references to the front-end ASIC and a precision clock conditioner for synchronization with the external timing system of the accelerator.

This detector as seen in Figure 6 is currently under production and will be soon available for various experiments at different accelerator facilities. One of the main applications of the 
KALYPSO detector is electro-optical spectral decoding (EOSD). This method allows to measure the longitudinal electron bunch profiles non-destructively on a shot-by-shot basis [6].

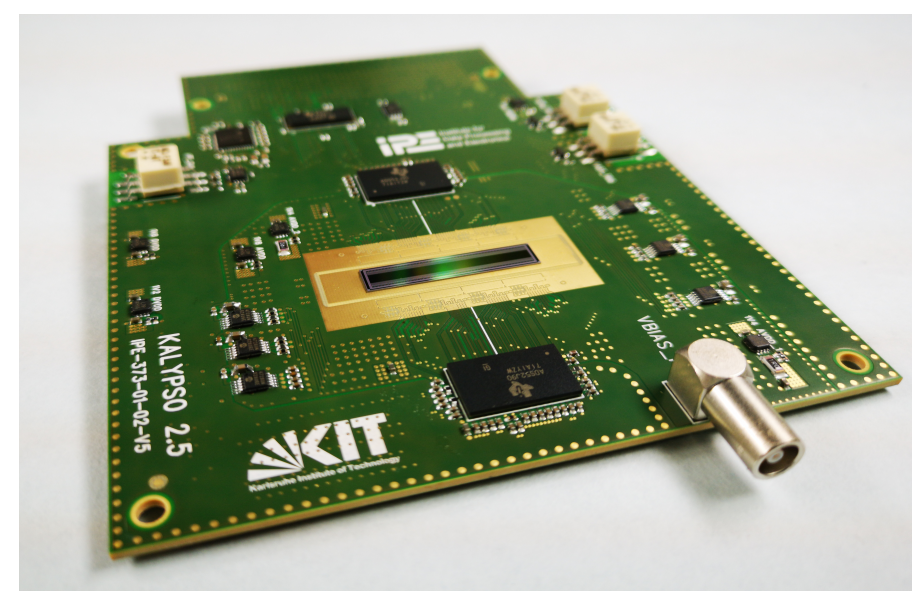

Figure 6: The Kalypso 2.5 board under final mounting

\section{Conclusion \& Future work}

The new version of KALYPSO is currently under development. This detector has been optimized for various wavelengths, i.e near infrared, visible and near ultra-violet. A new sensor technology is currently under design for an advanced transverse beam diagnostic detector system. This sensor will based on Low Gain Avalanche Detectors that offer a moderate internal gain while maintaining a low noise contribution [7]. The KALYPSO detector has opened up a new possibility to study fast beam dynamics in a synchrotron on a shot to shot basis at Mega frame rates.

\section{References}

[1] L. Rota, B.M. Balzer, M. Caselle, C. Gerth, N. Hiller, D.R. Makowski, et al., "KALYPSO: A Mfps Linear Array Detector for Visible to NIR Radiation", in Proc. IBIC2016, pp. 741-744, ISBN: 978-3-95450-177-9,

[2] Stefan Funkner, et al. "High throughput data streaming of individual longitudinal electron bunch profiles in a storage ring with single-shot electro-optical sampling,", in Physical Review Accelerators and Beams (PRAB) (2018), arXiv:1809.07530

[3] M. Caselle, et al. "Low-cost bump-bonding processes for high energy physics pixel detectors,", in JINST 11, C01050

[4] Sung-Mok Jung, et al. "Design and fabrication of multi-layer antireflection coating for III-V solar cell,”, in Current Applied Physics (2011), volume 11, pp.538-541

[5] A. Mozzanica, et al. "The gotthard charge integrating readout detector: design and characterization", in Journal of Instrumentation 7 (2012), C01019

[6] A. Borysenko, et al. "Electron bunch shape measurements using electro optical spectral decoding,", in Physics Procedia (2015), volume 77, pp.3-8

[7] H.F.-W.Sadrozinski, et al. "Sensors for ultra-fast silicon detectors,", in Nuclear Instruments and Methods in Physics Research (2014), Volume 765, pp.7-11 